Rev Biomed 2005; 16:273-280.

\title{
Activación de receptores dopaminérgicos por L-DOPA. De la acción terapéutica a las discinesias.
}

Revisión

Benjamín Florán-Garduñoํㅜ, Claudia Rangel-Barajas² .

Departamento de Fisiología, Biofísica y Neurociencias ${ }^{1}$, Sección Externa de Farmacología ${ }^{2}$, Centro de Investigación y de Estudios Avanzados del IPN, México, D.F., México.

\section{RESUMEN.}

La L-DOPA continúa siendo el tratamiento de elección en la enfermedad de Parkinson. En el presente trabajo se presentan y discuten las bases que explican el principal mecanismo farmacológico y que permiten entender su éxito terapéutico: el reemplazo de dopamina. Asimismo, se cuestiona el porqué de la generación de sintomatología colateral durante el tratamiento por reemplazo, principalmente la discinesia, y se citan las propuestas que con base en hallazgos experimentales recientes contribuirían a entenderla. Mecanismos adicionales que incluyen la activación directa de receptores dopaminérgicos y la existencia de un sistema de neurotransmisión DOPAérgico pudieran contribuir al efecto y formar parte de la explicación. Creemos que un mejor entendimiento de la acción de la L-DOPA en la enfermedad de Parkinson contribuirá al desarrollo de nuevas expectativas farmacológicas para su tratamiento. (Rev Biomed 2005; 16:273-280)
Palabras clave: L-DOPA, discinesia, enfermedad de Parkinson, receptores dopaminérgicos.

\section{SUMMARY.}

Activation of dopaminergic receptors by LDOPA. From therapeutics to dyskinesia.

L-DOPA is still the pharmacological treatment of choice for Parkinson disease. In the present work, we show and discuss the basis of the main mechanism of action of L-DOPA that explains its therapeutic effect: dopamine replacement. At the same time we question the generation of dyskinesia by a replacement mechanism and shown the experimental findings that help to understand it. Additional mechanisms including direct activation of dopamine receptors, and the existence of a DOPAergic system might contribute to the explanation. We believe that better understanding of the action of L-DOPA in Parkinson's disease will help in the development of new pharmacological treatments. (Rev Biomed 2005; 16:273-280)

Solicitud de sobretiros: Dr. Benjamín Florán-Garduño, Departamento de Fisiología, Biofísica y Neurociencias, CINVESTAV - IPN, Av. IPN No. 2508, San Pedro Zacatenco, México 07000, D.F.

Tel.: 5550613800 Ext. 5149 y 5137 Fax: 50613754 Correo electrónico: bfloran@fisio.cinvestav.mx

Recibido el 6/Abril/2005. Aceptado para publicación el 13/Octubre/2005.

Este artículo está disponible en http://www.uady.mx/sitios/biomedic/revbiomed/pdf/rb051647.pdf

Vol.16/No. 4/Octubre-Diciembre, 2005 


\section{B Florán-Garduño, C Rangel-Barajas.}

Key words: L-DOPA, dyskinesia, Parkinson's disease, dopaminergic receptors.

\section{Un poco de bases bioquímicas e historia.}

La DOPA (3,4-dihidroxifenilalanina) fue sintetizada por vez primera en 1911 y obtenida en el laboratorio como una mezcla racémica por Funk en 1911 (1). Aislada como L-DOPA en 1913, se consideró como una molécula biológicamente inactiva. Sin embargo, estudios de farmacocinética realizados años más tarde mostraron que la LDOPA causaba hiperglicemia y disminución de la presión sanguínea. Tales hallazgos marcaron el inicio formal del estudio de su actividad biológica y su papel en la síntesis de catecolaminas. Con el descubrimiento de Holtz en 1938 (1), la L-DOPA es considerada para el tratamiento en la Enfermedad de Parkinson (EP), ya que mostró que en el tejido hepático la L-DOPA era convertida a dopamina (DA), por acción de la enzima que se denominó descarboxilasa de la L-DOPA por Blaschko, en 1939 (1).

En 1961, la L-DOPA fue incorporada al tratamiento farmacológico de la EP y se observó un efecto benéfico en los síntomas de rigidez observados en esta enfermedad (2). Desde su introducción a la terapéutica, la L-DOPA o "dopa", como coloquialmente se le conoce, ha sido todo un reto para la farmacología moderna. La respuesta terapéutica es verdaderamente impactante. Una vez establecido el plan de tratamiento, la mejoría clínica es notoria, lo cual devuelve al paciente a las condiciones de vida que previamente tenía una vez que la enfermedad comenzó a minar su salud. Incluso, algunos autores sugieren el uso de la LDOPA con fines diagnósticos en diferentes formas de Parkinsonismo.

Sin embargo, el efecto que con aparente magia produce, tarde o temprano comienza a desaparecer y eventualmente los síntomas de la enfermedad retornan e incluso empeoran junto con la aparición de los efectos colaterales (3). El panorama del paciente es desalentador. ¿Qué es lo que ocurre? La respuesta a ciencia cierta no se conoce y algunas maniobras comienzan a efectuarse para controlar la situación: aumentar la dosis, variar la presentación y tiempo de administración, discontinuar su uso (mucho peor), coadministrar con otros medicamentos, etc. Desafortunadamente el problema de fondo no cambia.

Esta situación plantea nuevos retos para la terapéutica de la EP. Al margen del desarrollo de nuevas alternativas terapéuticas basadas en el entendimiento de la etiopatogenía y fisiopatología del padecimiento y de los ganglios basales (núcleos de afección por la enfermedad), la L-DOPA es en sí misma, un paradigma para la investigación. Del adecuado entendimiento de los mecanismos que subyacen tanto a sus efectos terapéuticos, como sus efectos colaterales y tóxicos, dependerá un mejor diseño de las nuevas drogas para el tratamiento farmacológico de la EP. En el presente trabajo pretendemos dar a conocer los nuevos puntos de vista sobre las bases farmacológicas de la acción de la L-DOPA, esto, basándose en hallazgos experimentales recientes y el impacto que pueden tener sobre la terapéutica.

\section{Enfermedad de Parkinson y terapia con L- DOPA.}

La degeneración y muerte de neuronas dopaminérgicas asociadas a la enfermedad de Parkinson representa el principio sobre el cuál se basa el uso de L-DOPA en su tratamiento. El razonamiento es simple: la muerte neuronal conlleva a una disminución de la dopamina en el núcleo estriado, principal sitio de acción del neurotransmisor, por lo tanto, al restaurar la dopamina resultará en una recuperación de la funcionalidad del sistema.

La administración directa de dopamina en los pacientes tiene sus inconvenientes por los efectos periféricos que produce (hipotensión aguda básicamente), así como en su rápido metabolismo, lo que produce bajas concentraciones en el cerebro. El precursor de la dopamina, la L-DOPA, resulta ser un mejor candidato $y$, aunque comparte

\section{Revista Biomédica}




\section{L-DOPA de la terapéutica a las discinesias.}

algunos de los efectos periféricos de la dopamina, éstos son con mucho menores. No obstante, permanece un efecto metabólico determinante: descarboxilación fuera del sistema nervioso central y degradación rápida ulterior. El uso de coadyuvantes que inhiben su metabolismo sólo en la periferia resuelve el problema. Los coadyuvantes resultan ser los inhibidores de la dopa-descarboxilasa hepática, tales como la carbidopa. La dopadescarboxilasa pertenece al grupo de enzimas conocidas como descarboxilasas de los aminoácidos aromáticos (AADC por sus siglas en inglés). Los inhibidores logran que se alcancen concentraciones importantes en cerebro lo que se supone la causa de su efecto terapéutico impresionante (4).

Una vez en el cerebro, el precursor debe ser convertido a dopamina y ejercer su efecto activando los receptores para el neurotransmisor. Esto constituye la base de lo se conoce como "mecanismo de acción por reemplazo" (5). En principio, esta hipótesis goza de lógica y apoyo experimental adecuado. Tanto en modelos de la enfermedad in vivo como in vitro han presentado datos experimentales que la apoyan (4, 6-7). El debate sobre los mecanismos de acción de la LDOPA en la EP, ahora radica en la respuesta a las siguientes preguntas:

1. ¿En dónde ocurre la descarboxilación de la L-DOPA para ser convertida a dopamina?

2. ¿Puede la descarboxilación producir suficiente dopamina para el reemplazo?

3. ¿Puede el reemplazo detener la progresión de la enfermedad?

4. ¿Por qué ocurren efectos colaterales de la L-DOPA, si el efecto se debe a la dopamina?

Con respecto al primer cuestionamiento, inicialmente se pensó que la L-DOPA era descarboxilada y convertida a dopamina en las neuronas remanentes (menos del 20\% del normal una vez que clínicamente se manifestó la enfermedad). El problema que representa esta suposición es que la cantidad de dopamina producida por estas neuronas sería poca, lo que llevaría a la segunda pregunta. Un hallazgo más que va en contra es esta idea, es que la muerte neuronal continúa a pesar del tratamiento (8), por lo tanto cada vez habría menor producción de dopamina. Los estudios basados en los modelos de Parkinson experimental, indican que la síntesis de dopamina alcanza cantidades importantes (9), particularmente en el estriado. La aparición rápida de dopamina en los líquidos de perfusión estriatales indican que potencialmente podría alcanzar el espacio sináptico y activar los receptores, lo que apoya la veracidad de la hipótesis del reemplazo.

A favor del reemplazo también esta el hecho de que la distribución de las AADC parece ser más amplia (10), tal que neuronas noradrenérgicas y serotoninérgicas pudieran ser un sitio alternativo de descarboxilación y fuente de dopamina para el reemplazo. Estas neuronas también contienen la enzima, pues recuérdese que la misma dopamina es un intermediario en la biosíntesis de otras catecolaminas, como la noradrenalina y la AADC, y participa en la síntesis de la serotonina (ver figura 1). Incluso se sabe que algunas fibras de las neuronas serotoninérgicas se redistribuyen aumentan sus axones en el estriado, como si existiera un mecanismo compensatorio al daño neuronal (11-13). Las fuentes alternativas producirían dopamina restaurando de cualquier manera la falta de ésta. No obstante, aún falta por demostrar que la dopamina sintetizada por esta u otras fuentes, alcanza el sitio de acción terapéutica, el estriado o mejor aún todos los ganglios basales. A este respecto en una revisión, Misu y sus colaboradores, en 1996 (14), expresan el concepto de lo que llaman el sistema L-DOPAérgico. Este sistema estaría representado por neuronas que expresan la AADC distribuidas en el cerebro y podrían ser la base del reemplazo.

Desde hace algún tiempo diversos autores han encontrado que la acción de la dopamina y por lo tanto, de la L-DOPA no sólo ocurre a nivel del estriado $(15-16,3,6)$, pues la inervación dopaminérgica (extraestriatal) llega prácticamente

Vol. 16/No. 4/Octubre-Diciembre, 2005 


\section{B Florán-Garduño, C Rangel-Barajas.}

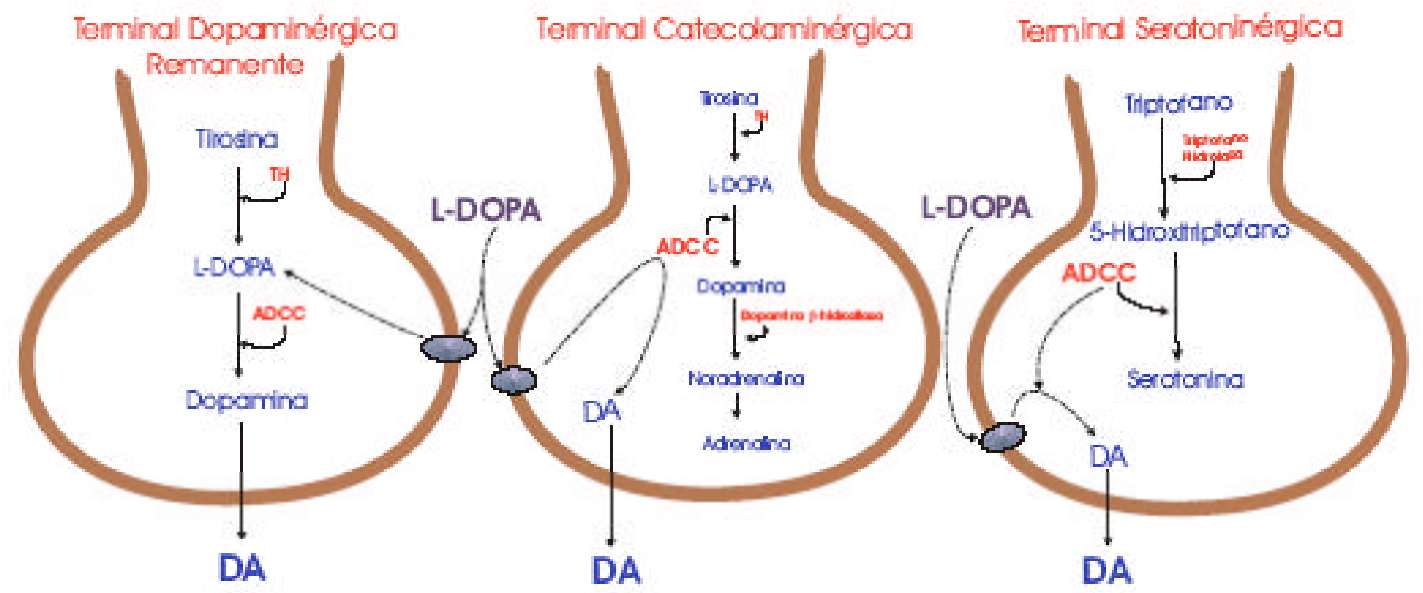

Figura 1.- Posibles sitios de síntesis de dopamina a partir de L-DOPA en terminales nerviosas del sistema nervioso central, durante la enfermedad de Parkinson.

a todos los núcleos que conforman a los ganglios basales, incluyendo al globo pálido, al núcleo subtalámico y a la misma sustancia nigra en su porción reticulada (17). La acción de la dopamina en estos sitios refuerza las acciones estriatales, además de regular el nivel de actividad cortical a través de las dos familias de los receptores dopaminérgicos, los D1 y los D2, mismos que se encuentran segregados en las neuronas de proyección del estriado. La activación de los receptores D1 estimula la actividad cortical por desinhibición, en tanto que la de D2 la refuerza bloqueando la actividad de los circuitos inhibitorios según el modelo de Penny y Young (18). Con estos hallazgos, cabe preguntarse si el reemplazo también ocurre en los núcleos extraestriatales, lo cuál no se conoce del todo.

¿Puede el reemplazo ser suficiente para detener la progresión de la enfermedad? El reemplazo no parece ser suficiente para detener la muerte neuronal. Estudios de resonancia magnética nuclear indican que la L-DOPA no detiene el proceso degenerativo, pues a pesar de la terapia el número de neuronas de la sustancia negra compacta, continúa disminuyendo (8). Esto indica que los mecanismos que provocaron la muerte neuronal no han cesado y pudieran inclusive continuar, como lo es el estrés oxidativo. Algunos estudios, en sus intentos por explicar la aparición de los efectos colaterales, indican que existe Revista Biomédica toxicidad de la L-DOPA que aceleraría la muerte de las neuronas por estrés oxidativo. Sin embargo esto sólo se ha mostrado in vitro, pero no in vivo (19). En consecuencia, si bien el reemplazo restaura la función, no es capaz de prevenir la muerte neuronal una vez que se ha iniciado el proceso degenerativo.

La aparición de efectos colaterales y secundarios en la terapia con L-DOPA representa el punto más difícil de la discusión. ¿Cómo es posible que la dopamina proveniente del reemplazo produzca los efectos secundarios (particularmente la discinecia)? ¿Es la L-DOPA o la dopamina la responsable de ellos?

Determinaciones de los efectos del tratamiento con L-DOPA sobre diferentes parámetros funcionales en Parkinson Experimental, como lo es la expresión de genes tempranos, la actividad motora, la actividad de las neuronas estriatales, etc., muestran que existen incrementos de la supersensibilidad funcional producida por la denervación o muerte de las neuronas. Este fenómeno está claramente asociado a la sobre actividad de receptores D1 (20, 21). Esto quiere decir que la terapia con L-DOPA incrementa aún más el proceso de supersensibilidad que la muerte neuronal inicio, un efecto que es difícil concebir que fuera provocado por la dopamina formada. En el modelo funcional propuesto por Penny y Young, para explicar la fisiopatología del Parkinson, los 


\section{L-DOPA de la terapéutica a las discinesias.}

movimientos involuntarios y exagerados, denominados discinéticos y observados en extremidades, cara y cuello, podrían explicarse por sobreactividad del circuito neuronal conocido como la vía directa, al cual están directamente asociados los receptores D1 $(22,23)$. Para explicar la asociación que implica el razonamiento anterior se podría pensar en efectos de la L-DOPA no atribuidos a conversión a dopamina, que discutiremos a continuación.

Efectos de la L-DOPA no atribuidos a su conversión en dopamina.

¿Un mecanismo de acción adicional al reemplazo podría ser responsable de los efectos colaterales de la L-DOPA? Diferentes líneas de investigación así lo sugieren. Se ha reportado en estudios de farmacocinética que existe una correlación directa entre elevadas concentraciones plasmáticas de L-DOPA y la aparición de las discinesias y es de notar que los efectos motores inducidos por la administración de L-DOPA no sólo se observan en modelos de parkinson experimental, sino también en animales intactos (9). Esto sugiere que los efectos adversos de la L-DOPA pudieran no solo depender de la supersensibilidad de los receptores a dopamina producidos por la muerte de las neuronas dopaminérgicas, sino también por efectos farmacológicos directos de la L-DOPA.

Diferentes estudios han reportado efectos de la L-DOPA por sí misma $(16,24-27)$ y existen dos maneras de explicarlas, las dos coinciden en que la L-DOPA tiene efectos per se, pero por mecanismos diferentes (figura 2).

La primera propuesta le asigna a la L-DOPA el papel de neuromodulador y/o neurotransmisor. Esta idea se inicia con trabajos como los de Goshima en 1991 (28), en los que mostró que la L-DOPA inhibe la liberación de dopamina y facilita la de noradrenalina vía la activación de receptores $\beta$-adrenérgicos. Estos efectos no son bloqueados por la inactivación de la AADC ni mimetizados por sus metabolitos. Evidencias más recientes han llevado a proponer la existencia de un sistema

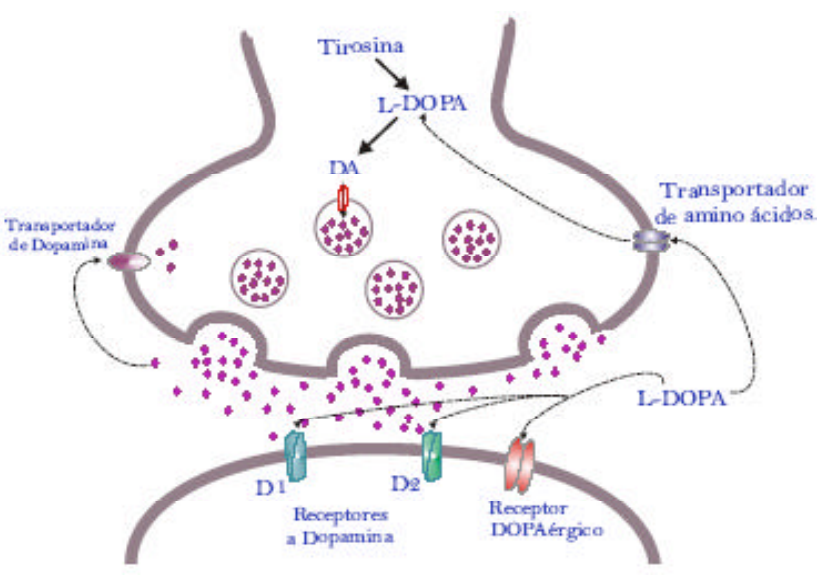

Figura 2.- Mecanismos de acción adicionales de la LDOPA en la enfermedad de Parkinson, por conversión a dopamina o por activación de receptores.

DOPAérgico basándose en que cumple con varios criterios como son: síntesis, metabolismo, transporte activo y liberación inducida farmacológica o fisiológicamente $(14,29)$. El principal sitio donde este sistema pudiera existir es el núcleo del tracto solitario (30), región en la que se ha mostrado por inmunohistoquímica la presencia de neuronas tirosina-hidroxilasa positivas, L-DOPA positivas y AADC negativas (14). También se ha reportado que en el hipocampo, en el estriado y en la médula espinal, la estimulación eléctrica produce liberación de L-DOPA. La primera función atribuible a este sistema seria el control del reflejo baroreceptor. En la revisión de Misu del 2002 (29), estos efectos están ampliamente discutidos. La idea de un sistema así es atractiva, sin embargo, desde nuestra perspectiva tiene dos aspectos que resolver. Uno es que la evidencia clara del receptor DOPAérgico no existe ni por estudios de receptor-ligando específicos, ni por estudios de clonación. Por otro lado, habría que explicar como pueden surgir efectos colaterales a la administración de L-DOPA como las discinesias, tratándose se un sistema funcional. Mayores estudios deberán hacerse al respecto.

En una segunda propuesta, los efectos de la administración de L-DOPA son atribuidos a la activación directa de los receptores D1 y D2. 


\section{B Florán-Garduño, C Rangel-Barajas.}

Estudios farmacológicos en presencia de inhibidores de la AADC, han mostrado efectos de la L-DOPA mediados por la activación de los receptores D1 (16), así como D2 en ratas normales (26), tratadas con reserpina (27) y con Parkinson experimental (31). A este respecto, tampoco existe evidencia directa de estudios de unión receptor-ligando específica. Interesantemente, Newman-Tancredi y colaboradores (32) han mostrado unión de baja afinidad en el receptor D4 de humano transfectado a células de ovario de hamster. Sin embargo, esta evidencia no es suficiente y requiere mayor investigación, pero abre una posibilidad más.

Ahora bien de acuerdo a la pregunta inicial; ¿Puede la L-DOPA, activando receptores a dopamina producir sus efectos colaterales? Esto también amerita investigación más amplia, aunque algo pudiera especularse. Una hipótesis sobre la generación de las discinesias, sugiere hiperactividad de la vía directa de los ganglios basales a la cual están asociados los receptores D1 (23). Los receptores D1 al ser activados de manera normal producen hiperactividad y conducta estereotipada (33). Consecuentemente, podría suponerse que la L-DOPA generaría las discinesias activando los receptores D1 de manera directa e incrementando su supersensibilidad provocada por la denervación. Apoyando esta hipótesis, datos de nuestro laboratorio no publicados aún, han mostrado que el tratamiento con L-DOPA en ratas con denervación dopaminérgica incrementa aún más la supersensibilidad del receptor D1 presináptico de las terminales estriado-nigrales. También hallazgos como los de Aceves y colaboradores (16), Nakazato y Akiyama (7) han mostrado que en ratas lesionadas del sistema dopaminérgico con 6hidroxidopamina, la L-DOPA y su metabolito la 3-metoxitiramina (3-MT), activan a los receptores D1/D5 de manera directa, cumpliendo así con los requisitos para la generación de las discinesias. Así, reiteramos que continuar investigando al respecto es sumamente importante para entender las acciones farmacológicas de la L-DOPA.

\section{Conclusión.}

En resumen, los efectos farmacológicos de la L-DOPA en el tratamiento de la enfermedad de Parkinson parecen ir más allá de los inicialmente descritos en el mecanismo por reemplazo. La explicación de los efectos secundarios generados por la administración de L-DOPA requiere nuevas explicaciones y base experimental para sustentarlas. Dos nuevas propuestas sobre los mecanismos de acción de la L-DOPA, parecen comenzar a dar luz de lo que probablemente este ocurriendo. La primera sugiere que la L-DOPA constituye en realidad un sistema de neurotransmisión en los ganglios basales. La segunda, sugiere que la LDOPA tiene efectos per se, es decir, no mediados por su conversión a dopamina y que implican la activación de receptores dopaminérgicos. Esta activación directa de los receptores llevaría a una sobreactividad de las vías neurales del control motor y explicaría la aparición de las discinesias. Investigar más sobre estos puntos, podría generar ideas para nuevos fármacos con las propiedades de la L-DOPA, pero sin los efectos no deseados.

\section{REFERENCIAS.}

1.- Hornykiewicz O. L-DOPA: from a biologically inactive amino acid to a successful therapeutic agent. Amino Acids 2002; 23:65-70.

2.- Barbeu A, Murphy GF, Sourkes TL. Excretion of dopamine in diseases of basal ganglia. Science 1961; 133:1706-7.

3.- Bezard E, Brotchie JM, Gross CE. Pathophysiology of levodopa-induced dyskinesia: potential for new therapies. Nat Rev Neurosci 2001; 2:577-88.

4.- Dluzen D, Reddy A, McDermott J. The aromatic amino acid decarboxylase inhibitor, NSD-1015, increases release of dopamine: response characteristics. Neuropharmacology 1992; 31:1223-9.

5.- Hornykiewicz O. The mechanisms of action of L-dopa in Parkinson's disease. Life Sci 1974; 15:1249-59.

\section{Revista Biomédica}




\section{L-DOPA de la terapéutica a las discinesias.}

6.- Lopez A, Munoz A, Guerra MJ, Labandeira-Garcia JL. Mechanisms of the effects of exogenous levodopa on the dopamine-denervated striatum. Neuroscience 2001; 103:639-51.

7.- Nakazato T, Akiyama A. Behavioral activity and stereotypy in rats induced by L-DOPA metabolites: a possible role in the adverse effects of chronic L-DOPA treatment of Parkinson's disease. Brain Res 2002; 930:13442.

8.- Whone AL, Watts RL, Stoess AJ, Davis M, Reske S, Nahmias C, et al. Slower progression of Parkinson's disease with ropinirole versus levodopa: The REAL-PET study. Ann Neurol 2003; 54:93-101.

9.- Pearce RK, Heikkila M, Linden IB, Jenner P. L-DOPA induces dyskinesia in normal monkeys: behavioural and pharmacokinetic observations. Psychopharmacology (Berl) 2001; 156:402-9.

10.- Ikemoto K. Significance of human striatal D-neurons: implications in neuropsychiatric functions. Prog Neuropsychopharmacol Biol Psychiatry 2004; 28:429-34.

11.- Luthman J, Bolioli B, Tsutsumi T. Verhofstad A, Jonsson G. Sprouting of striatal serotonin nerve terminals following selective lesions of nigro-striatal dopamine neurons in neonatal rat. Brain Res Bull 1987; 19:269-74.

12.- Tanaka H, Kannari K, Maeda T, Tomiyama M, Suda $\mathrm{T}$, Matsunaga M. Role of serotonergic neurons in LDOPA-derived extracellular dopamine in the striatum of 6-OHDA-lesioned rats. Neuroreport 1999; 10:631-4.

13.- Maeda T, Kannari K, Shen H, Arai A, Tomiyama M, Matsunaga M, et al. Rapid induction of serotonergic hyperinnervation in the adult rat striatum with extensive dopaminergic denervation. Neurosci Lett 2003; 343:1720.

14.- Misu Y, Goshima Y, Ueda H, Okamura H. Neurobiology of L-DOPAergic systems. Prog Neurobiol 1996; 49:41554.

15.- Boraud T, Bezard E, Guehl D, Bioulac B, Gross C. Effects of L-DOPA on neuronal activity of the globus pallidus externalis (GPe) and globus pallidus internalis (GPi) in the MPTP-treated monkey. Brain Res 1998; 787:157-60.
16.- Aceves J, Floran B, Martinez-Fong D, Sierra A, Hernandez S, Mariscal S. L-DOPA stimulates the release of. Neurosci Lett 1991; 121:223-6.

17.- Cossette M, Levesque M, Parent A. Extrastriatal dopaminergic innervation of human basal ganglia. Neurosci Res 1999; 34:51-4.

18.- Penney JB, Young AB. Speculations on the functional anatomy of basal ganglia disorders. Annu Rev Neurosci 1983; 6:73-94.

19.- Mytilineou C, Walker RH, JnoBaptiste R, Olanow CW. Levodopa is toxic to dopamine neurons in an in vitro but not an in vivo model of oxidative stress. J Pharmacol Exp Ther 2003; 304:792-800.

20.- Chartoff EH, Marck BT, Matsumoto AM, Dorsa DM, Palmiter RD. Induction of stereotypy in dopaminedeficient mice requires striatal D1 receptor activation. Proc Natl Acad Sci U S A 2001; 98:10451-6.

21.- Gerfen CR, Miyachi S, Paletzki R, Brown P. D1 dopamine receptor supersensitivity in the dopaminedepleted striatum results from a switch in the regulation of ERK1/2/MAP kinase J Neurosci 2002; 22:5042-54.

22.- Gerfen CR, Engber TM, Mahan LC, Susel Z, Chase TN, Monsma F, et al. D. R. D1 and D2 dopamine receptorregulated gene expression of striatonigral and striatopallidal neurons. Science 1990; 250:1429-32.

23.- Aubert I, Guigoni C, Hakansson K, Li Q, Dovero S, Barthe N, et al. Increased D1 dopamine receptor signaling in levodopa-induced dyskinesia. Ann Neurol 2005; 57:1726.

24.- Nakazato T, Akiyama A. Effect of exogenous L-dopa on behavior in the rat: an in vivo voltammetric study. Brain Res 1989; 490:332-8.

25.- Nakamura S, Yue JL, Goshima Y, Miyamae T, Ueda H, Misu Y. Non-effective dose of exogenously applied Ldopa itself stereoselectively potentiates postsynaptic D2 receptor-mediated locomotor activities of conscious rats. Neurosci Lett 1994; 170:22-6.

26.- Yue JL, Nakamura S, Ueda H, Misu Y. Endogenously released L-dopa itself tonically functions to potentiate postsynaptic D2 receptor-mediated locomotor activities of conscious rats. Neurosci Lett 1994; 170:107-10. 


\section{B Florán-Garduño, C Rangel-Barajas.}

27.- Fisher A, Biggs CS, Eradiri O, Starr, M. S. Dual effects of L-3,4-dihydroxyphenylalanine on aromatic L-amino acid decarboxylase, dopamine release and motor stimulation in the reserpine-treated rat: evidence that behaviour is dopamine independent. Neuroscience 2000; 95:97-111.

28.- Goshima Y, Nakamura S, Misu Y. Ldihydroxyphenylalanine methyl ester is a potent competitive antagonist of the L-dihydroxyphenylalanineinduced facilitation of the evoked release of endogenous norepinephrine from rat hypothalamic slices. J Pharmacol Exp Ther 1991; 258:466-71.

29.- Misu Y, Goshima Y, Miyamae T. Is DOPA a neurotransmitter? Trends Pharmacol Sci 2002; 23:262-8.

30.- Yue JL, Okamura H, Goshima Y, Nakamura S, Geffard M, Misu Y. Baroreceptor-aortic nerve-mediated release of endogenous L-3,4-dihydroxyphenylalanine and its tonic depressor function in the nucleus tractus solitarii of rats. Neuroscience 1994; 62:145-61.

31.- Treseder SA, Rose S, Jenner P. The central aromatic amino acid DOPA decarboxylase inhibitor, NSD-1015, does not inhibit L-DOPA-induced circling in unilateral 6OHDA-lesioned-rats. Eur J Neurosci 2001; 13:162-70.

32.- Newman-Tancredi A, Audinot V, Chaput C, Verriele L, Millan MJ. [35S]Guanosine-5'-O-(3-thio) triphosphate binding as a measure of efficacy at human recombinant dopamine D4.4 receptors: actions of antiparkinsonian and antipsychotic agents. J Pharmacol Exp Ther 1997; 282:18191.

33.- O'Sullivan GJ, Roth BL, Kinsella A, Waddington JL. SK\&F 83822 distinguishes adenylyl cyclase from phospholipase C-coupled dopamine D1-like receptors: behavioural topography. Eur J Pharmacol 2004; 486:27380. 